\title{
Le COVID-19, miroir grossissant du problème carceral
}

\author{
Yves Cartuyvels \\ Professeur ordinaire, Université-Saint-Louis-Bruxelles
}

C'est un plaisir et un honneur que de participer à cet ouvrage en hommage à Sonja Snacken. Sonja, ce n'est un secret pour personne, est une des meilleures spécialistes de la prison en Belgique et en Europe. Juriste dévoyée - ce sont les meilleurs -, elle s'est très rapidement orientée vers une approche sociologique du droit et de l'institution carcérale. Membre du comité de rédaction de la revue Déviance et Société, cette parfaite trilingue a fait profiter la revue de son expertise précieuse, avec un calme et un sourire jamais démentis. Elle a aussi largement contribué à maintenir en Belgique des liens entre les criminologues néerlandophones et francophones et les collaborations avec Kristel Beyens et Jenneke Christiaens, notamment, lui doivent beaucoup. Je lui suis particulièrement reconnaissante d'avoir maintenu ce fil et ouvert des portes entre ces deux mondes.

La prison n'est pas mon premier objet de recherche. Dans cette brève contribution, je vais pourtant tenter quelques réflexions sur cette institution, sorte de prolongement d'un questionnement développé il y a 20 ans dans un texte intitulé à l'époque 'le dilemme des prisons'. Prenant appui sur le crise récente du COVID-19 et son impact sur le régime carcéral, je voudrais questionner son remplacement souhaitable ou probable, au croisement d'une tension entre abolitionnisme et réductionnisme pénal.

\section{La prison, le noyau dur de la peine moderne}

La prison s'est imposée comme la peine de référence en Occident à la fin du XVIIIe siècle. Succédant au 'spectacle punitive' de l'Ancien Régime,

1 Y. Cartuyvels, Le dilemme des prisons, Revue Nouvelle, nº4, 1999, pp. 22-37. 
elle s'impose dans le fil d'un double discours humaniste et utilitariste. Humaniste, dès lors qu'il s'agit de mettre fin à la cruauté ritualisée de peines corporelles pour des raisons d'humanité (mais aussi par crainte que le 'spectacle punitive' ne finisse par se retourner contre le souverain) ${ }^{2}$. Utilitariste, car la prison devient le relais idéal d'une pénalité de calcul en quête d'efficacité, fondée sur un double pari: la plus grande dissuasion d'une peine certaine et proportionnée à la gravité du délit d'une part; la plus grande effectivité d'une peine modérée que les jurés hésiteront moins à prononcer qu'une peine spectaculaire mais disproportionnée en regard de l'acte commis. D'entrée de jeu, la prison apparaît socialement plus efficace et utile que les peines corporelles antérieures ou que d'autres peines comme la peine de mort ou la déportation. Promoteur du panoptisme carcéral, Jeremy Bentham le souligne très clairement: la silhouette noire des prisons situées en périphérie ou à l'intérieur des villes impressionne plus le corps social que l'expulsion du criminel et sa relégation dans des îles lointaines où le souvenir même de son existence finit par disparaître ${ }^{3}$.

Très rapidement, la prison se voit également associer un idéal de correction: assumant l'héritage de la pénitence chrétienne, la peine privative de liberté doit contribuer à réformer l'âme du condamné pour en refaire un citoyen ou une force de travail au service de la société. A quelques rares exceptions près, le détenu apparait ici comme un citoyen conditionnel, momentanément privé de ses droits et de sa liberté, destiné à réintégrer le monde social après avoir purgé sa peine. Dès ses débuts, la prison est associée au correctionnalisme.

Si l'efficacité dissuasive de la prison pour le grand public est par définition difficile à évaluer, son potentiel de prévention spéciale, tout comme son idéal de correction, s'avèrent rapidement des échecs. Tout au long du XIXe siècle, les réformateurs de la prison s'empoignent sur les meilleurs moyens de favoriser une prison correctrice, capable d'amender et de resocialiser les condamnés. Défenseurs du modèle Pensylvanien contre partisans du modèle Auburnien aux Etats-Unis, Alexis de Tocqueville contre Charles Lucas en France ${ }^{4}$, Ducpétiaux versus Prins en Belgique ${ }^{5}$, la querelle des modèles fait

2 Voy. M. Foucault, Surveiller et punir. Naissance de la prison, Paris, Gallimard, 1975, pp. 64-66.

3 J. Bentham, Panopticon versus New-South Wales, London, Baldwin, 1812.

4 F. Digneffe, "Problèmes sociaux et représentations du crime et du criminel. De Howard (1777) à Engels (1845) ", in Ch. Debuyst, Fr. Digneffe, J.M. Labadie, A.P. Pires, Histoire des savoirs sur le crime \& la peine, Bruxelles, Montréal, Ottawa, De Boeck Université, PUO, PUM, 1995, pp. 180-190.

5 Voy. A. Ducpetiaux, Réforme des prisons. Système cellulaire, Bruxelles, 1865, 
rage: faut-il privilégier un régime fondé sur l'isolement cellulaire, propice au retour réflexif sur soi et plus économe en termes de maintien de l'ordre carcéral, mais aussi susceptible de plonger certains dans la folie? Ou favoriser un régime commun, éventuellement progressif, plus apte à préparer le retour dans la vie sociale, même s'il rend plus difficile la gestion interne de la prison et favorise des contacts criminogènes problématiques une fois le détenu rendu à la liberté? Derrière le conflit d'approches, une réalité: la prison, lieu d'humanisation de la peine, ne remplit pas les fonctions qui lui sont formellement assignées: instrument de gestion des illégalismes des classes populaires - ces 'classes dangereuses' dont la menace se précise avec le siècle -, la prison est un lieu de non droit, marqué par l'arbitraire et les rapports de force; loin de diminuer les taux de criminalité, son usage nourrit la récidive; espace de violence quotidien, portée par un temps vide que rien ou si peu ne vient remplir, elle enfonce le condamné dans une culture de la déviance bien plus qu'elle réforme les âmes.

\section{Répondre à l'échec de la prison: réformes et peines alternatives}

La fin du XIXe siècle pénal est marquée dans le monde occidental par un vent de réforme, sous l'impulsion du mouvement de la défense sociale. Une idée force s'impose: sérier le régime punitif en fonction de la dangerosité du délinquant. En Belgique, Adolphe Prins, père de la défense sociale, est porteur de ce projet qui suppose de classer et de trier les délinquants. Si les plus dangereux (les récidivistes, les aliénés délinquants) doivent pouvoir rester en détention au-delà du régime d'une peine classique, les moins dangereux (délinquants primaires) doivent par contre pouvoir quitter la prison avant l'exécution totale de leur peine, voire éviter la peine de prison ${ }^{6}$. Adossé au premier pôle d'une logique de bifurcation pénale, le correctionnalisme débouche ainsi sur l'introduction d'une loi sur la libération conditionnelle et les condamnations conditionnelles en 1888. C'est la réponse au constat proféré tout au long du siècle: la prison n'amende pas, elle ne traite pas, elle ne resocialise pas. Plus de temps on y reste, plus de chances on a d'y revenir. Pour ceux qui sont le moins englués dans la délinquance ou qui apparaissent

reprint in Déviance et Société, 12, 1, pp. 29-55 et A. Prins, La loi sur la libération conditionnelle, Journal des Tribunaux, 1888, n 555 et sv.

6 Ibidem. Ce thème revient dans de nombreux ouvrages d'Adolphe Prins. 
comme moins dangereux, il vaut mieux en sortir avant le terme de la peine, quitte à faire l'objet d'une surveillance allongée au dehors.

Face à ce constat d'échec de la prison, d'autres initiatives sont prises dans la deuxième moitié du XXe siècle et au début du XXIe pour désengorger l'institution carcérale. C'est le temps des 'alternatives', dans les années 1960 et suivantes: en Belgique, loi sur la suspension, le sursis et la probation, alternatives à la détention préventive (une grande partie des détenus ne sont pas des condamnés mais des prévenus en attente de jugement), alternatives aux poursuites (médiation pénale, travail d'intérêt général au stade du parquet) et peines alternatives (peine de travail, peine de probation autonome, peine de surveillance électronique) se succèdent. En même temps, s'élabore un droit de l'exécution de la peine et une 'juridictionnalisation' de son contrôle pour réintroduire plus de droit et diminuer l'arbitraire dans l'exécution de la peine.

Mais rien n'y fait: les alternatives ont beau se multiplier, elles restent des 'alternatives' qui, à défaut d'être érigées en peine de référence unique pour certaines infractions, ont bien du mal à mordre sur le réflexe carcéral7. Malgré les réformes régulières de la détention préventive, malgré la diversification des peines, malgré les multiples modalités d'aménagement de l'exécution de la peine, les prisons restent surpeuplées. De même, le durcissement des conditions d'octroi des aménagements de peine ou la construction de nouvelles prisons demeurent le premier réflexe pour répondre à des peurs diverses, pas nécessairement justifiées par une augmentation des chiffres de la délinquance enregistrée, plus régulièrement liées à la médiatisation d'un fait divers spectaculaire. La prison reste la place forte d'un archipel punitif qui, aujourd'hui, prend aussi très largement appui sur des dispositifs de contrôle hors les murs ${ }^{8}$.

\section{Le COVID-19 en prison: révélateur d'un problème structurel ancien}

Autrefois, les murs de la prison devaient effrayer et dissuader. Aujourd'hui, ils sont censés nous rassurer contre la menace que représente les condamnés.

7 Voy. Y. Cartuyvels, Ch. Guillain, Th. Slingeneyer, The development of alternative sanctions in the Belgian context: introduction, in S. BERNARDI (dir.), Prison overcrowding and alternative sanctions in Europe: European sources and national legal systems, Napoli, Casa Ed. Jovene, 2016, pp. 116-121.

8 Sur ce point, voy. F. Mc NEIL, K. Beyens, Offender supervision in Europe, New York, Palgrave Macmillan, 2013. 
Autrefois, la prison était assumée comme peine unique. Aujourd'hui, elle n'est censée intervenir qu'en 'dernier recours'. A deux siècles d'écart, deux discours différents. Mais une réalité identique: sous l'emprise d'un 'populisme pénal' qui ne désarme pas, la peine de prison reste la référence, que seule peut-être la surveillance électronique sera un jour en mesure de contester: mais d'une peine privative de liberté dans les murs à une peine privative de liberté hors les murs, c'est toujours d'une peine privative de liberté qu'il s'agit. La privation de liberté ne fait que s'ajuster à l'hybridation progressive entre des sociétés disciplinaires à vocation normalisatrices et des sociétés de contrôle à dominante gestionnaire. Sur ce point, Michel Foucault avait raison: la prison est bien le réflecteur puissant de tendances sociales plus larges et l'engouement pour la surveillance électronique, association d'une logique de confinement à domicile et de tracing, est sans doute révélatrice de la croissance d'une logique de contrôle virtuel à laquelle nous allons tous être sommés d'adhérer.

Pour l'heure, nous n'y sommes pas encore. Aujourd'hui, à l'heure du COVID-19, la population carcérale reste importante et est touchée de plein fouet. En Belgique, les prisons sont encore et toujours surpeuplées. Les conditions de détention s'aggravent et portent clairement atteinte aux droits fondamentaux des détenus, déjà secoués ces derniers mois par les grèves du personnel pénitentiaire. Les soins sont lacunaires et cette question, qui n'est pas neuve', prend une résonnance particulière. Et si la situation est grave pour les détenus, elle ne l'est pas moins pour le personnel pénitentiaire appelé, plus que jamais, à travailler dans des conditions précaires. A cet égard, la crise du COVID-19 ne fait rien surgir de neuf: elle révèle au grand jour les conditions de précarité que connaissent les divers acteurs de l'univers carcéral ${ }^{10}$.

La prise de position du Conseil central de surveillance pénitentiaire, le 30 mars 2020, est éclairante ${ }^{11}$. Rappelant la situation critique dans les prisons face à l'épidémie, le Conseil souligne la nécessité, d'aménager, à l'intérieur des prisons, le régime de la détention pour répondre à la crise. Mais il demande aussi de limiter au maximum la population carcérale. Vu le risque de crise sanitaire et sécuritaire (le confinement, pratiquement impossible en prison, est aussi source de tensions et d'émeutes possibles), le Conseil

9 https://www.atsp.be/appel/

10 Y. Cartuyvels, O. Nederlandt, M. Neve, La prison face au covid19: zoom sur un angle mort de la démocratie, Chronique Carta Academica, Le Soir, mise en ligne le 30 mars 2020 (La prison face au covid\#1192593)

11 Communiqué du Conseil central de surveillance pénitentiaire, 30 mars 2020 (http://oipbelgique.be/fr/?p=1097). 
estime qu'il est aujourd'hui 'urgent de réduire drastiquement le nombre de personnes détenues', en limitant strictement les nouvelles incarcérations et en assouplissant les conditions de sortie. Libérer les personnes en détention préventive, surseoir à l'exécution des peines d'emprisonnement, recourir de manière accrue aux alternatives à l'emprisonnement, anticiper la libération des personnes susceptibles d'être libérées avant terme, libérer provisoirement pour raisons médicales les personnes les plus vulnérables ou encore octroyer une grâce collective font partie des leviers mobilisables à cet effet.

\section{Profiter de la crise pour repenser la place de la prison: abolir ou réduire?}

En matière de politique de peines comme dans d'autres domaines, la crise du COVID-19 peut déboucher sur deux voies.

Soit, il s'agira au plus vite de refermer la parenthèse en estimant que 'ceci n'est pas une crise' mais un accident de parcours et que le plus urgent sera de continuer après comme avant. On continuera alors à construire de nouvelles prisons, en sachant - ceci est une très vieille loi en matière pénitentiaire - que l'offre conditionne la demande et que ces nouvelles prisons, une fois construites, seront très rapidement saturées à leur tour, appelant inexorablement la construction de nouveaux établissements. A l'intérieur des murs, on continuera à faire l'impasse sur un droit à la santé et une véritable politique de soins pour les détenus, alors même que la loi de principe concernant l'administration des établissements pénitentiaires et le statut juridique des détenus du 12 janvier 2005 prône un traitement équivalent en matière de santé dans et hors les murs de la prison.

Soit, la mesure est prise qu'il s'agit d'une vraie crise, de celles qui mettent en pleine lumières des anomalies structurelles qui nous empêchent, en ce domaine comme dans d'autres, de penser et de fonctionner après comme avant. Ceci reviendrait alors à questionner fondamentalement la place et le fonctionnement de la prison comme mode de contrôle social dans des sociétés démocratiques fondées sur le droit. C'était, en des termes pratiquement inchangés, la question abordée il y a 20 ans dans le texte consacré au 'dilemme des prisons' évoqué ci-dessus:

d'un côté, chercher à faire de la prison un espace de droit, c'est entériner l'existence d'un espace carcéral qui souffre d'un grave déficit de légitimité, accepter le maintien d'un système pénal qui repose sur une privation de liberté qu'on peut juger contraire aux droits de l'homme, accepter 
la violence d'une logique discriminatoire qui gère l'exclusion $(. .$.$) ; de$ l'autre, refuser toute réforme de la prison, c'est s'empêcher de penser le développement d'un droit de l'exécution des peines, source de garanties, de protection et d'améliorations véritables pour les détenus confrontés, dans leur vie quotidienne, à l'arbitraire et au vide du monde carceral'. ${ }^{12}$

Faut-il abolir ou réformer la prison? La posture abolitionniste a eu son heure de gloire (théorique) dans les années $1970^{13}$. Prônant l'abandon du droit pénal au nom des effets individuels, sociaux et politiques, de la logique même de la peine, ce courant s'est largement construit sur une critique radicale de la prison pour dénoncer le pénalo-centrisme de nos sociétés contemporaines. L'abolitionnisme propose un changement de vocabulaire ('tort', 'conflit' ou encore 'situations problems' plutôt qu'infraction) et le remplacement du recours à la pénalité par des modes 'civils' ou communautaires de règlement des conflits. Assez logiquement, les abolitionnistes devraient refuser toute réforme de la prison, dès lors que toute réforme est prisonnière de la 'bouteille à mouche', selon l'expression de $\mathrm{P}$. Watzlawick ${ }^{14}$, tant qu'elle ne va pas dans le sens d'une déconstruction radicale de l'institution.

Cette posture, qui a sa cohérence intellectuelle, est difficile à soutenir en pratique pour, me semble-t-il, deux raisons. La première est qu'elle contraint à faire un choix entre l'idéal d'un projet et le réel d'un monde vécu et à faire primer le premier sur le second. Pour le dire autrement, comme le souligne bien G. Chantraine, l'abolitionnisme fait de la 'Cause' à défendre quelque chose 'de plus important que la situation immédiate et concrète de ceux (derrière les barreaux) que la Cause prétend défendre, créant ainsi une autre réalité idéologique non moins pernicieuse que celle à laquelle elle prétendait s'opposer'15. Un certain nombre d'acteurs abolitionnistes ne se résolvent d'ailleurs pas à cette posture qui les amènerait, par exemple, à refuser toute intervention éducative en prison puisqu'une telle intervention revient à légitimer l'institution dont ils dénoncent l'existence. Ces acteurs tiennent alors un discours un peu schyzophrénique, défendant dans le discours une posture dont leur engagement concret dans les prisons est la négation.

12 Y. CaRtuyvels, Réformer ou supprimer: le dilemme des prisons, in O. de Schutter, D. Kaminski (eds.), L'institution du droit pénitentiaire, Paris, LGDJ, 2002, pp. 130-131.

13 L. Hulsman, Peines perdues. le système pénal en questions, Paris, Le Centurion, 1982.

14 P. Watzlawick, La mouche et la bouteille à mouches, in P. Watzlawick (ED.), L'invention de la réalité. Contributions au constructivisme, Paris, Seuil, Gallimard, 1988, pp. 269-276.

15 G. Chantraine, Prison et regard sociologique. Pour un décentrage de l'analyse critique, Champ pénal/Penal field, 2004, vol. I, p. 4. 
La seconde raison est que si l'abolitionnisme déroule une logique implacable dans sa déconstruction de la prison et sa critique de la logique de la peine, il s'avère beaucoup plus hésitant et nettement moins convainquant dans sa phase de 'reconstruction': Est-il possible pour tous les actes ou comportements actuellement criminalisés d'éviter le recours à un mode de contrôle social fondé sur la contrainte? Et si oui, par quoi remplacer le pénal, ses institutions et ses acteurs?

Sur le premier point, les abolitionnistes affrontent (ou n'affrontent pas) un problème classique en droit, qui est celui 'des cas difficiles': comment mobiliser, par exemple un règlement 'alternatif', civil ou communautaire, fondé sur la discussion ou la persuasion, lorsqu'une des parties 'en conflit' est dans le déni, dans le refus de toute forme de négociation ou dans la conviction, pour une raison ou l'autre, qu'il a son droit ou sa morale pour lui? On pense, par exemple, aux criminels de guerre nazis qui, en 1946 à Nuremberg, ont plaidé non coupable, ne reconnaissant pas la portée criminelle de leurs actes. Sur quoi va-t-on négocier? On pourrait bien évidemment trouver d'autres exemples.

Sur le second, l'abolitionnisme repose à mon sens sur une illusion, héritage plus ou moins inconscient d'un impensé marxiste. Plus prolixe que Marx sur cette question Engels expliquait que la plupart des crimes étaient le produit de la 'guerre de tous contre tous' générée par le capitalisme et sa logique d'exploitation et que l'avènement d'une société sans classes ayant renonçé à la propriété privée des moyens de production amènerait la disparition naturelle de la plupart des crimes. Dans une société communiste qui a 'substitué la paix sociale à la guerre sociale' et 'où l'on met l'accent sur la source du crime', seuls demeurent de rares 'conflits civils', qui seront 'facilement résolus par des arbitres'. La plupart des institutions pénales deviennent en fait 'superflues', au point que 'la justice concernée par les cas criminels cesse d'elle même' ${ }^{16}$. Pour Engels, une société communiste égalitaire et débarrassée de la lutte des classes produit donc naturellement l'abolition de la justice pénale. Tout comme elle annonce aussi - thème largement développé par Marx - le dépérissement de l'Etat (auquel succède le règne de la 'Gemeinwesen', un régime politique marqué par l'auto-régulation

16 F. ENGELs, 'Speech in Elberfeld, 8 février 1845', Rheinische Jahrbücher zur gesellschaftlichen Reform, 1845, bd.I, pp. 5-6 (https://marxists.catbull.com/archive/marx/ works/1845/02/15.htm) 
de la communauté) ${ }^{17}$ et le dépérissement du droit (instrument par définition de domination bourgeoise $)^{18}$.

Vue à deux siècles de distance, l'analyse de Marx et Engels sur ce point apparaît clairement réductrice: certes, une part importante des comportements criminalisés peut toujours être considérée comme le produit d'un modèle de société fondé sur l'exploitation et les inégalités. Il suffit de regarder la population des prisons pour se convaincre de la pertinence de cette grille de lecture sociale du crime. Mais, il paraît difficile de réduire tous les comportements criminalisés à cette seule 'guerre' et d'en inférer qu’avec une société égalitaire, le crime et les appareils de contrôle social étatique disparaitront, point sur lequel Engels demeure d'ailleurs prudent ${ }^{19}$. Comme l'a bien perçu le sociologue français E. Durkheim, pour des raisons qui tiennent à son mode de structuration symbolique, toute société érige toujours des actes en déviance, fut-ce 'une société de saints ${ }^{20}$, ce qui pose inexorablement la question de sa gestion. Or, en matière de gestion des déviances, les sociétés modernes ont horreur du vide. Comme l'a bien montré Michel van de Kerchove, un processus de décriminalisation laisse ainsi très rapidement place à l'avènement d'autres modes de contrôle social ${ }^{21}$. On ne peut dès lors échapper à cette question du 'remplacement', qui amène 'à décrypter la reconfiguration, hors les murs, des différents types de contrôles sociaux formels et informels, qui n’auraient peut-être rien à envier à l'abominable' prison ${ }^{22}$.

Cette reconfiguration ne débouche pas nécessairement sur des exemples enthousiasmants. A la fin du XIXe siècle, la criminologie positiviste était d'une certaine manière abolitionniste: pas du côté des infractions, volet sur lequel elle adoptait une posture 'réaliste', ne remettant pas en question le

17 F. ENGELS, Lettre à Auguste Bebel (18-25 mars 1875), in K. Marx, Critique du programme de Gotha, Paris, Les éditions sociales, 2008, 98-99. Voy, sur ce point, Y. Douet, Le problème du dépérissement du droit chez Marx et Engels, Droit \& Philosophie, 10, 2018 (http://droitphilosophie.com/upload/files/pdf/dp10-t03_douet. pdf).

18 K. Marx, Commentaires en marges du programme du Parti ouvrier allemand, in K. Marx, Critique du programme de Gotha, op. cit., 59-60.

19 Engels évoque une disparition de "la plupart de activités des corps judiciaires et administratifs ». Au XXe siècle, les sociétés officiellement sans classes, l'URSS et la Chine communiste, n'ont par ailleurs aucunement renoncé à criminaliser des comportements " antisociaux », se caractérisant en outre par des régimes répressifs puissants.

20 E. Durkheim, Les règles de la méthode sociologique, Paris, P.U.F., 1973, p. 68.

21 M. van de KERCHOVE, " Médicalisation » et " fiscalisation » du droit pénal: deux versions asymétriques de la dépénalisation, Déviance et Société, 1981, 5, 1, pp. 1-23.

22 Idem, p.4, note 19. 
caractère de construction sociale (et donc relatif) des incriminations pénales. Mais bien du côté de la peine, qu'elle estimait sans pertinence à l'égard d'un individu déterminé par sa nature et/ou son milieu. Pourquoi chercher à adresser par la peine un message moral à un individu fondamentalement irresponsable? Mais la contrepartie, on le sait, sera dans ce projet 'alternatif au pénal' porté par l'Ecole positive italienne de substituer à la logique de la peine des mesures de soin et de sécurité potentiellement nettement plus contraignantes et arbitraires au nom des impératifs du contrôle social. La dépénalisation programmée, entendue ici au sens d'une sortie de la logique punitive, se solde par l'adoption d'un modèle de contrôle social non punitif certes, mais pas moins coercitif pour autant. Dépénaliser n'est aucunement en soi un gage de moins de répression. On sait aussi, pour prendre un autre exemple, que certaines 'situations problématiques' aux confins de l'étiquetage criminel sont gérées de manière administrative, générant un 'droit pénal de l'ombre' dont la violence n'a rien à envier à celle du droit pénal proprement dit. Au point d'avoir amener la Cour Européenne des droits de l'homme à considérer parfois que certaines de ces matières faisaient partie de la 'matière pénale' et devaient bénéficier, dans leur traitement, des garanties associées au pénal. C’est embêtant, mais oui, parfois, le pénal est aussi protecteur.

Sauf à s'enfermer dans un 'angélisme exterminateur'23, les abolitionnistes ne peuvent faire l'économie de cette question du remplacement. Ils ne peuvent plus non plus, à mon sens, se contenter de répéter les pétitions de principe issues de textes optimistes écrits dans les années 1970 ou 1980: l'époque n'est plus la même, les rapports entre l'Etat et la société ont changé et la place faite aux communautés dans de sociétés fragmentées également. Quels seraient, aujourd'hui, dans des sociétés néo-libérales soucieuses avant tout de dérégulation et de privatisation, les effets d'un appel exclusif à des modèles non étatiques et non contraignants de gestion des comportements étiquetés comme déviants? Quel impact dans nos sociétés du XXIe siècle, productrices de populisme et de replis communautaires à forte connotation identitaires? Face à 'la communauté qui vient'24, est-on sûr de vouloir mettre à bas le recours à l'Etat et ses institutions pénales et recourir, toujours et pour tout, à des 'arbitres' divers comme l'évoquait Engels? Est-on sur de vouloir privilégier dans tous les cas des modes civils ou communautaires de règlement des conflits, fondés sur la 'persuasion' et la

23 Je reprends cette expression au titre d'un ouvrage consacré aux évolutions sécuritaires de nos sociétés contemporaines, publié dans les années 1990 (A.G. Slama, L'angélisme exterminateur: essai sur l'ordre moral contemporain, Paris, Hachette, 1993.

24 G. Agamben, La Communauté qui vient. Théorie de la singularité quelconque, Paris, Seuiol, 1990. 
douce violence d'un consensus au sein de la communauté? Toutes les 'parties en conflit' y trouveraient-elles leur compte? On peut en douter, par exemple lorsqu'un arbitrage dans la communauté traduirait un point de vue culturel clairement orienté par des valeurs défavorables à une des parties en conflit ${ }^{25}$. Face à ces questions, les abolitionnistes contemporains semblent hésiter et on les comprend ${ }^{26}$.

Si l'auteur de ces lignes n'est pas abolitionniste, il est par contre fondamentalement partisan d'une éthique réductionniste en matière de prisonisation et de recours au pénal. Il faut réduire drastiquement le recours à la peine privative de liberté, qu'elle se situe dans ou en dehors les murs; ériger d'autre peines non privatives de liberté en sanction principale et non plus uniquement en 'peine alternative'; favoriser une logique réparatrice là où c'est possible, tout le monde a à y gagner. Et il faut s'interroger sérieusement sur les profils socio-économiques de la population pénale. Marx et Engels étaient loin d'avoir tout faux et il reste urgent de tirer les conséquences politiques d'un modèle de développement social qui, aux antipodes de tous les appels à la solidarité entendus ces dernières semaines en contexte de crise COVID-19, crée inégalités et précarité, discrimination et criminalisation.

\section{Conclusion}

La prison est au cœur d'un modèle de surveillance disciplinaire dont elle est l'archétype. C'est tout l'intérêt de l'analyse de M. Foucault de l'avoir montré, en ne se focalisant pas tant sur la prison pour la prison que sur la prison en tant que révélateur de mécanismes de pouvoir et de domination propres aux

25 Sur ce point, on lira avec intérêt une contribution de K. Lecoyer consacrée à la médiation informelle réalisée par des Imans en matière de divorce en Belgique (K. Lecoyer, 'Internormative Family Conflict 'Mediation' within Belgian Muslim Families', Revue Interdisciplinaire d'Etudes Juridiques. Droit en contexte, 2020 (à paraître).

26 Lors d'une conférence récente sur le thème abolitionnisme et féminisme qui s'est tenue à l'Université Saint-Louis-Bruxelles, en décembre 2020, G. Ricordeau témoignait de cette difficulté. Soulignant d'entrée de jeu, comme pour s'en dédouaner, la difficulté de construire une posture abolitionniste présentée comme toujours " work in progress ", l'auteure reconnaissait que, "dans certains cas..., sans doute, il était légitime qu'une 'victime' s'adresse à la police ", que dans d'autres, il serait difficile de " persuader l'auteur »... ou encore que "si ce dernier (l'auteur) restait dans le déni, le poids du groupe pourrait le contraindre à évoluer "... Outre le recours étonnant dans le chef d'une abolitionniste aux catégories pénales d'auteur et de victime, on retombe ici sur une posture plus classiquement réductionniste (voy aussi. G. Ricordeau, Pour elles toutes. Femmes contre la prison, Chico, Lux éditeur, 2019). 
sociétés modernes, actifs de manière plus visible dans son enceinte que dans d'autres champs de la vie sociale.

Instrument marqué par un seuil d'inhumanité particulièrement important, la prison doit être combattue. Ce combat suppose de réfléchir à sa place (à réduire) comme mode de contrôle social et à son évolution (en termes de droits) dans des sociétés régies par le rule of law. Mais il nous impose également d'envisager la transformation probable de la privation de liberté telle qu'elle est en train de s'accélérer dans nos sociétés de contrôle. A cet égard, la crise du COVID-19 marque sans doute un saut important. L'engouement pour les systèmes de tracking divers que suscitent les peurs sanitaires, lié à une logique de confinement à domicile dont nous mesurons le poids aujourd'hui, souligne une mutations technologique majeure qui questionne nos modes de vie et notre rapport aux libertés. Dans le 'meilleur des mondes' qui s'annonce, la privation de liberté pourrait bien muter rapidement sous l'effet des 'progress' technologiques, laissant entrevoir des souffrances et des atteintes aux droits d'une nature nouvelle. 